\title{
Impact of tourniquet during total knee arthroplasty when tranexamic acid was used: a meta-analysis of randomized controlled trials
}

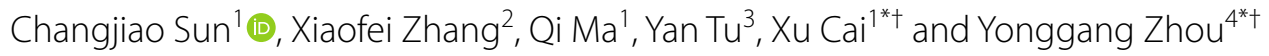

\begin{abstract}
Introduction: The efficacy of tourniquet use during primary total knee arthroplasty (TKA) is thought to reduce intraoperative blood loss, improve surgical exposure, and optimize cement fixation. Tranexamic acid (TXA) use can decrease postsurgical blood loss and transfusion requirements. This review aimed to appraise the effects of tourniquet use in TKA for patients with tranexamic acid use.

Methods: A meta-analysis was conducted to identify relevant randomized controlled trials involving TXA plus a tourniquet (TXA-T group) and use of TXA plus no tourniquet (TXA-NT group) in TKA. Web of Science, PubMed, Embase, Cochrane Controlled Trials Register, Cochrane Library, Highwire, CNKI, and Wanfang database were searched from 2010 through October 2021.

Results: We identified 1720 TKAs (1690 patients) assessed in 14 randomized controlled trials. Compared with the TXA-NT group, the TXA-T group resulted in less intra-operative blood loss $(P<0.00001)$ and decreased duration of surgery $(P<0.00001)$, however more hidden blood loss $(P=0.0004)$ and less knee range of motion $(P<0.00001)$. No significant differences were found between two groups in terms of decrease in hemoglobin $(P=0.84)$, total blood loss $(P=0.79)$, transfusion rate $(P=0.18)$, drainage volume $(P=0.06)$, Visual Analogue Scale (VAS) at either the day of surgery $(P=0.2), 1$ day $(P=0.25), 2$ day $(P=0.39), 3$ day $(P=0.21), 5$ day $(P=0.21), 7$ day $(P=0.06)$ or 1 month after surgery $(P=0.16)$, Hospital for Special Surgery (HSS) score at either 7 day $(P=0.10), 1$ month $(P=0.08), 3$ month $(P=0.22)$ or 6 month after the surgery $(P=0.92)$, Knee circumference $(P=0.28)$, length of hospital $(P=0.12)$, and complications such as intramuscular venous thrombosis $(P=0.81)$, deep venous thrombosis $(P=0.10)$, superficial infection $(P=0.45)$, deep wound infection $(P=0.64)$, and delayed wound healing $(P=0.65)$.
\end{abstract}

Conclusion: No big differences could be found by using or not tourniquet when use the TXA, though some benefits are related to operation time and less intra-operative blood loss by using tourniquet and TXA, Using the tourniquet was related to more hidden blood loss and less knee range of motion. More adequately powered and betterdesigned randomized controlled trials (RCTs) studies with long-term follow-up are required to validate this study.

\footnotetext{
*Correspondence: suncjdoctor@163.com; zhouyonggang2021@126.com

${ }^{+} \mathrm{Xu}$ Cai and Yonggang Zhou have contributed equally to this work and

should be considered co-Corresponding author.

1 Department of Orthopedic, Beijing Tsinghua Changgung Hospital,

School of Clinical Medicine, Tsinghua University, No. 168 Litang Road,

Dongxiaokou Town, Changping District, Beijing 102218, China

${ }^{4}$ Department of Orthopaedic Surgery, The First Medical Centre, Chinese

PLA General Hospital, No. 28 Fuxing Road, Beijing 100853, China

Full list of author information is available at the end of the article
} original author(s) and the source, provide a link to the Creative Commons licence, and indicate if changes were made. The images or other third party material in this article are included in the article's Creative Commons licence, unless indicated otherwise in a credit line to the material. If material is not included in the article's Creative Commons licence and your intended use is not permitted by statutory regulation or exceeds the permitted use, you will need to obtain permission directly from the copyright holder. To view a copy of this licence, visit http://creativecommons.org/licenses/by/4.0/. The Creative Commons Public Domain Dedication waiver (http://creativecommons.org/publicdomain/zero/1.0/) applies to the data made available in this article, unless otherwise stated in a credit line to the data. 
Keywords: Tranexamic acid, Blood loss, Total knee arthroplasty, Tourniquet, Randomized controlled trials

\section{Introduction}

Tourniquet use has been considered an essential element of the total knee arthroplasty (TKA). Many surgeons apply a tourniquet during TKA to reduce blood loss and operative times, improve surgical exposure, optimize cement fixation, and increase tissue concentrations of antibiotic drugs through intraosseous regional administration [1-5]. However, the once highly regarded advantages of tourniquet use have come under great scrutiny in light of its potential disadvantages. Issues which bring its use into question included reperfusion injury [6], patellar tracking issues [7], increased perioperative pain [8, 9], increased postoperative limb swelling [10,11], decreased postoperative range of motion (ROM) [12], delayed rehabilitation [12], increased risk of thrombosis [13, 14], more frequent wound complications [15-17], and its negative effect on patients with vascular disease [18]. More recently, as a new strategy for reducing blood loss, perioperative administration of tranexamic acid (TXA) has gained popularity during TKA, mitigating some of the adverse effects of tourniquet use. Several studies have confirmed that TXA significantly reduces blood loss and transfusion requirements without increasing venous thrombotic events [19-21]. Although there are many systematic reviews and meta-analysis comparing tourniquet use and non-tourniquet use during TKA, there was no meta-analysis comparing the effects of TXA plus a tourniquet and the use of TXA plus no tourniquet. Therefore, we compare the impact of TXA plus a tourniquet and use of TXA plus no tourniquet in TKA. This review aimed to appraise the effects of tourniquet use in TKA for patients with tranexamic acid use.

\section{Methods}

\section{Protocol and registration}

The study protocol was registered with International prospective register of systematic reviews (PROSPERO), and the registration number was CRD42020185403. This meta-analysis was performed using a predetermined protocol following the Preferred Reporting Items for Systematic Reviews and Meta-Analyses (PRISMA) statement to assess the results' quality to make sure our meta-analysis's results reliable and veritable.

\section{Search strategy}

A meta-analysis was conducted to identify relevant randomized controlled trials involving TXA plus a tourniquet (TXA-T group) and use of TXA plus no tourniquet (TXA-NT group in TKA. Web of Science, PubMed,
Embase, Cochrane Controlled Trials Register, Cochrane Library, Highwire, CNKI, and Wanfang database were searched from 2010 through October 2021. The keywords used were "total knee replacement," "total knee arthroplasty," "tourniquet," "tranexamic acid," "TXA," "randomized controlled trials" in conjunction with Boolean operators "AND" or "OR." We used Review Manager Software for MAC to perform the meta-analysis.

\section{Inclusion criteria}

Studies were eligible if (1) the intervention was patients undergoing primary TKA using TXA and a tourniquet (TXA-T group); (2) the comparator was patients undergoing primary TKA using TXA and without tourniquet use (TXA-NT group); (3) the design of the study was a randomized controlled trial (RCTs); (4) the clinical outcome data were intra-operative blood loss (IBL), hidden blood loss (HBL), total blood loss (TBL), drainage volume, decrease in hemoglobin level, transfusion rate, Visual Analogue Scale (VAS) score, Hospital for Special Surgery (HSS) score, knee circumference, knee range of motion (ROM), length of stay (LOH), complications including intramuscular venous thrombosis (IMVT), deep venous thrombosis (DVT), superficial infection, deep wound infection, delayed wound healing. (5) The studies were required to contain at least one clinical outcome data; the exclusion criteria were as follows: (1) observational studies; (2) non-RCTs; (3) studies with insufficient clinical outcome data.

\section{Data extraction process}

Two reviewers (C.J.S and Q.M.) used a standardized form to extract data. A third reviewer (X.C) was used to resolve disagreements in eligibility, data extraction, or quality assessment. Extracted data included the primary data based on the following: first author, year of publication, participants, age, gender, body mass index, diagnosis, anesthesia, prothesis, patellar resurfacing, tourniquet pressure, tourniquet realizing time, TXA administration, drainage, thromboprophylaxis.

\section{Assessment of studies}

The studies' methodological quality was assessed following the instructions in the Cochrane Handbook for Systematic Reviews of Interventions.

\section{Statistical Analysis}

RevMan software (version 5.4; The Cochrane Collaboration) was used for the analysis. The statistical 
heterogeneity was tested with the $X^{2}$ test and $I^{2}$ test. $I^{2}<25 \%$ was considered low statistical heterogeneity, $I^{2}<50 \%$ moderate statistical heterogeneity, and $I^{2}<75 \%$ high statistical heterogeneity. If the $P$ value of heterogeneity was less than 0.1 , heterogeneity would exist. Then, the random-effects model was used for meta-analysis. Data were summarized as the ratio of relative risk (transfusion rate, complications including the rate of IMVT, DVT, superficial infection, deep wound infection, delayed wound healing.) or the difference between means (IBL, HBL, TBL, drainage volume, decrease in hemoglobin level, VAS score, HSS score, knee circumference, knee ROM and LOH). For studies that did not report standard deviations (SDs), it was calculated from $P$ values, confidence intervals, or standard errors. The results were considered as a statistically significant difference when $P$ values were less than 0.05 .

\section{Results}

The search strategy identified 259 studies, of which 245 were excluded after screening in Fig. 1. The literature search identified 259 citations. Of these, 164 duplicates were removed. After examining the titles and abstracts of the 95 remaining articles, we excluded 77 papers according to the inclusion and exclusion criteria; the full text of 18 articles was retrieved. Because we could not acquire sufficient data in one article, and four studies were non-RCTs. Hence, four studies were excluded. Fourteen articles were assessed for eligibility. In Palanne's [22] article, there were two subgroups comparing TXA + tourniquet group with TXA + NT group. One is the spinal anesthesia subgroup, The other is the general anesthesia subgroup. So we divided the study into two groups: Palanne 2020 (1) and Palanne 2020 (2). Finally, we identified 1720 TKAs (1690 patients) assessed in 15 randomized controlled trials [2, 22-34]. Study baseline characteristics and general intervention information are summarized in Tables 1, 2, 3 and 4.

The risk of bias summary and bias graph for RCTs is shown in Figs. 2 and 3. Fourteen studies adequately described the correct randomization. Thirteen studies demonstrated sufficient allocation concealment. Four studies described the blinding of participants and personnel. No studies described the blinding of outcome assessment. All thirteen articles retained complete outcome data and avoided selective reporting. We rated as unclear risk of other bias because we cannot ignore other potential dangers of biases. As a result, there is low or moderate risk of bias in most of the articles reviewed (Fig. 2).

\section{Blood loss}

Nine RCTs reported IBL; three RCTs reported HBS and seven RCTs reported total blood loss. The pooled data showed that the TXA with tourniquet group had significantly decreased IBL $\quad(M D=-109.89,95 \%$ CI [ $148.04,-71.74], P<0.00001$, Fig. 4). However, the TXA without tourniquet group has significantly increased HBL $(\mathrm{MD}=117.64,95 \%$ CI [52.4, 182.88], $P=0.0004$, Fig. 4). Both groups experienced similar TBL $(\mathrm{MD}=7.13,95 \%$ CI $[-46.23,60.49], P=0.79$, Fig. 4).

\section{Drainage volume}

Five RCTs reported drainage volume. The forest plot showed that the drainage volume was not significantly different between the two groups ( $M D=69.50,95 \% \mathrm{CI}$ $[-3.91,142.9], P=0.06$, Fig. 5).

\section{Decrease in hemoglobin}

Four RCTs reported a decrease in hemoglobin. The pooled data revealed that the reduction in hemoglobin was not significantly different between the two groups $(\mathrm{MD}=7.90,95 \% \mathrm{CI}[-5.44,6.68], P=0.84$, Fig. 6).

\section{Transfusion rate}

Seven RCTs reported the transfusion rate. The forest plot revealed that the transfusion rate was not significantly different between the two groups $(\mathrm{RD}=0.07$, $95 \%$ CI $[-0.02,0.04], P=0.18$, Fig. 7 ).

\section{Duration of surgery}

Five RCTs reported duration of surgery, TXA with tourniquet group have significantly decreased time of surgery compared with TXA-NT group $(\mathrm{MD}=-1.05$, $95 \%$ CI $[-1.46,-0.64], P=<0.00001$, Fig. 8$)$.

\section{VAS}

Four RCTs reported VAS on the day of surgery. Ten RCTs reported VAS on the first day after surgery. Six RCTs reported VAS on the third day after surgery. Two RCTs reported VAS on the second and fifth day after surgery. Three RCTs reported VAS on the seventh day after surgery. Two RCTs reported VAS at 1 month after surgery. The results of random-effects metaanalysis showed no significant differences between the two groups in the postoperative VAS score at either the day of surgery $(\mathrm{MD}=1.56,95 \% \mathrm{CI}[-5.0,3.62]$, $P=0.20$, Fig. 9), first day ( $\mathrm{MD}=0.42,95 \% \mathrm{CI}[-0.29$, 1.13], $P=0.25$, Fig. 9), second day $(\mathrm{MD}=0.16,95 \% \mathrm{CI}$ $[-0.21,0.54], P=0.39$, Fig. 9), third day $(\mathrm{MD}=0.20$, 95\% CI $[-0.12,0.53], P=0.21$, Fig. 9), fifth day $(\mathrm{MD}=0.95,95 \% \mathrm{CI}[-0.52,2.42], P=0.21$, Fig. 9), 


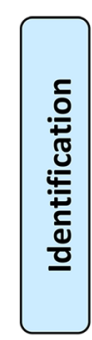

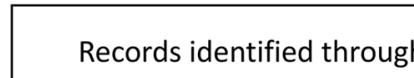
database searching $(n=259)$
Additional records identified through other sources $(n=0)$
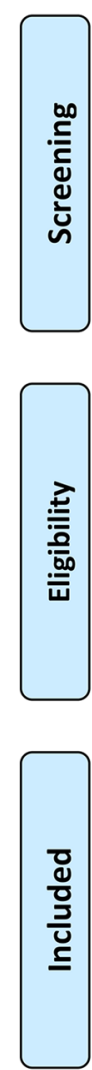

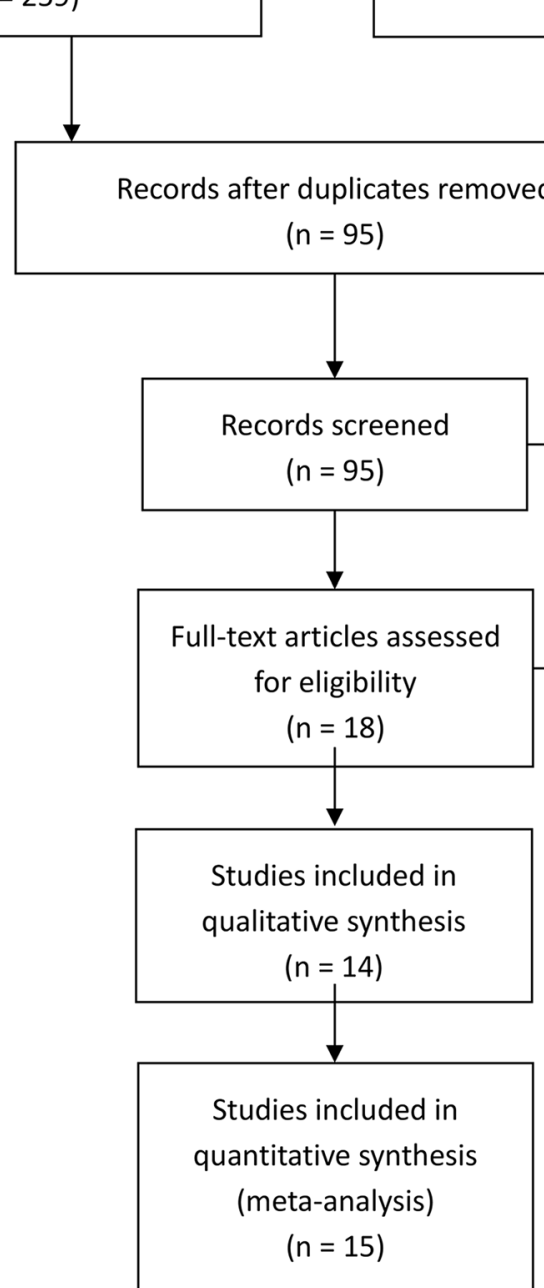

Fig. 1 The search results and selection procedure. The literature search identified 259 citations. Of these, 164 duplicates were removed. After examining the titles and abstracts of the 95 remaining articles, we excluded 77 papers according to the inclusion and exclusion criteria; the full text of 18 articles was retrieved. Fourteen articles were assessed for eligibility. In Palanne's article, there were two subgroups comparing TXA + tourniquet group with TXA + NT group. So we divided the study into two groups. Finally, we identified 1720 TKAs (1690 patients) assessed in 15 randomized controlled trial

seventh day $(\mathrm{MD}=0.89,95 \% \mathrm{CI}[-0.04,1.83], P=0.06$, Fig. 9)or 1 month after surgery $(\mathrm{MD}=0.16,95 \% \mathrm{CI}$ $[-0.06,0.39], P=0.16$, Fig. 9).

\section{HSS}

Three RCTs reported HSS 7 day, 1 month, 3 month after surgery. Two RCTs reported HSS 6 month after surgery. The pooled results showed that both groups experienced similar HSS scores at either 7 day $(\mathrm{MD}=-10.11,95 \% \mathrm{CI}$ $[-21.98,1.76], P=0.10$; Fig. 10), 1 month $(\mathrm{MD}=-2.93$,
95\% CI [-6.22, 0.35], $P=0.08$; Fig. 10), 3 month $(\mathrm{MD}=-0.73,95 \% \mathrm{CI}[-1.89,0.43], P=0.22$; Fig. 10) or 6 month after the surgery $(\mathrm{MD}=-0.08,95 \% \mathrm{CI}[-1.84$, 1.67], $P=0.92$; Fig. 10).

\section{Knee circumference}

Two RCTs reported knee circumference. We detected a similar knee circumference between two groups $(\mathrm{MD}=5.86,95 \% \mathrm{CI}-4.72,16.44], P=0.28$; Fig. 11$)$. 
Table 1 The detailed baseline characteristics information

\begin{tabular}{|c|c|c|c|c|c|c|}
\hline \multicolumn{7}{|c|}{ The detailed baseline characteristics information } \\
\hline Author/year & Patients & Knees & Mean age (years) & Female gender (\%) & BMI & Diagnosis \\
\hline \multicolumn{7}{|c|}{ Tourniquet use with TXAVNo tourniquet use with TXA } \\
\hline Alexandersson 2018 & $38 / 43$ & $38 / 43$ & $68 / 69.7$ & $52.6 / 48.8$ & 28.6/27.9 & $380 \mathrm{~A} / 43 \mathrm{OA}$ \\
\hline Concina 2019 & $50 / 50$ & $50 / 50$ & NA & NA & NA & NA \\
\hline Ejaz 2014 & $33 / 31$ & $33 / 31$ & $68 / 68$ & $45.5 / 45.2$ & $25 / 25$ & $330 A / 310 A$ \\
\hline Huang 2017 & $50 / 50$ & $50 / 50$ & $66.2 / 65.1$ & $64 / 68$ & $25.1 / 24.4$ & $500 A / 500 A$ \\
\hline Ma 2017 & $31 / 32$ & $31 / 32$ & $66.8 / 67.2$ & $61.3 / 65.6$ & $24.38 / 24.02$ & $310 A / 320 A$ \\
\hline Palanne $2020^{1}$ & $101 / 99$ & 101/99 & $64 / 63$ & $72.3 / 58.6$ & $30.7 / 30.8$ & 1010A/990A \\
\hline Palanne $2020^{2}$ & $99 / 96$ & $99 / 96$ & $63 / 65$ & $62.6 / 61.5$ & $30.5 / 29$ & $99 \mathrm{~A} / 960 \mathrm{~A}$ \\
\hline Wang 2017 & 30 & $30 / 30$ & $65.9 / 65.9$ & $86.7 / 86.7$ & $26.6 / 26.6$ & $300 A / 300 A$ \\
\hline Wang 2019 & $30 / 30$ & $30 / 30$ & $62.8 / 64.1$ & $90 / 73.3$ & $23.48 / 23.68$ & $300 A / 300 A$ \\
\hline Xie 2017 & $45 / 45$ & $45 / 45$ & $66.2 / 66.1$ & $85 / 75$ & $26.1 / 25.9$ & NA \\
\hline Xue 2018 & $30 / 30$ & $30 / 30$ & $68.2 / 69.1$ & $60 / 53.3$ & NA & $300 A / 300 A$ \\
\hline Yu 2017 & $40 / 40$ & $40 / 40$ & $60.65 / 62.6$ & NA & NA & $400 A / 400 A$ \\
\hline Zak 2021 & $161 / 166$ & $161 / 166$ & $66.5 / 67.6$ & $57 / 66$ & $30.55 / 30.63$ & 1610A/1660A \\
\hline Zeng 2021 & $50 / 50$ & $50 / 50$ & $68.44 / 68$ & $84 / 86$ & $25.34 / 26.13$ & $500 A / 500 A$ \\
\hline Zhou 2017 & $72 / 68$ & $72 / 68$ & $66.8 / 69.1$ & $81.9 / 89.7$ & $26.1 / 25.7$ & 500A/52OA; 22RA/16RA \\
\hline
\end{tabular}

The detailed baseline characteristics information, including the number of TKAs, age, gender, BMI, and two groups' diagnosis

$\mathrm{OA}$, osteoarthritis; RA, rheumatoid arthritis; BMI, body mass index; TXA, tranexamic acid

Table 2 The detailed information about surgery

\begin{tabular}{|c|c|c|c|c|}
\hline \multicolumn{5}{|c|}{ The detailed information of surgery } \\
\hline Author/year & Anesthesia & Prothesis & $\begin{array}{l}\text { Patellar } \\
\text { resurfacing }\end{array}$ & Drainage \\
\hline Alexandersson 2018 & Spinal/general anesthesia, & NexGen fixed bearing (Zimmer) & No & No \\
\hline Concina 2019 & NA & Triathlon ${ }^{\circledR}$ (Stryker) and Attune ${ }^{\circledR}$ (DePuy) & NA & No \\
\hline Ejaz 2014 & Spinal anesthesia & NexGen fixed bearing (Zimmer) & Yes & No \\
\hline Huang 2017 & General anesthesia & NA & NA & Yes \\
\hline Ma 2017 & General anesthesia and FNB & PS, PFC (DePuy) & NA & Yes \\
\hline Palanne $2020^{1}$ & Spinal anesthesia & Triathlon ${ }^{\circledR}$ (Stryker) & Yes & No \\
\hline Palanne $2020^{2}$ & General anesthesia & Triathlon ${ }^{\circledR}$ (Stryker) & Yes & No \\
\hline Wang 2017 & General anesthesia & Genesisll (Smith\&Nephew) or NexGen (Zimmer) & No & Yes \\
\hline Wang 2019 & General anesthesia & PS Haixing (Weihai) & NA & Yes \\
\hline Xie 2017 & General anesthesia & PS (DePuy) & No & Yes \\
\hline Xue 2018 & General anesthesia & NA & No & Yes \\
\hline Yu 2017 & Spinal anesthesia & NA & Yes & Yes \\
\hline Zak 2021 & NA & NA & NA & No \\
\hline Zeng 2021 & & PS, PFC (DePuy) & NA & Yes \\
\hline Zhou 2017 & General anesthesia & PS, PFC (DePuy) & NA & Yes \\
\hline
\end{tabular}

The detailed information of surgery including anesthesia, prosthesis, patellar resurfacing, and drainage of two groups

FNB, femoral nerve block; PS, posterior cruciate-stabilizing; CR, cruciate retaining

\section{Knee ROM}

Six RCTs reported knee ROM. TXA with tourniquet group has significantly decreased knee ROM compared with TXA-NT group $(\mathrm{MD}=-2.68,95 \%$ CI $-3.30,-2.07], P<0.00001$; Fig. 12).
$\mathrm{LOH}$

Nine RCTs reported LOH. No significant difference was found for $\mathrm{LOH}$ between both groups $(\mathrm{MD}=0.40$, 95\% CI - 0.1, - 0.9], $P=0.12$; Fig. 13). 
Table 3 The detailed information of tourniquet use

\begin{tabular}{|c|c|c|c|}
\hline Author/year & Tourniquet pressure & Tourniquet realizing Time & TXA administration \\
\hline Alexandersson 2018 & $300 \mathrm{mmHg}$ & After bandage applied & Intravenously, 1 g, 10 min before surgery \\
\hline Concina 2019 & $300 \mathrm{mmHg}$ & Before wound closure & $\begin{array}{l}\text { Intravenously, } 15 \mathrm{mg} / \mathrm{kg}, 20 \mathrm{~min} \text { before surgery and } \\
\text { after } 4 \mathrm{~h}\end{array}$ \\
\hline Ejaz 2014 & $250 \mathrm{mmHg}$ & After bandages applied & Orally, $1 \mathrm{~g}$, before surgery; orally, $0.5 \mathrm{~g} 3 \mathrm{~h}$ after surgery \\
\hline Huang 2017 & $100 \mathrm{~mm} \mathrm{Hg}$ above systolic pressure & NA & $\begin{array}{l}\text { Intravenously, } 20 \mathrm{mg} / \mathrm{kg}, 5 \text { to } 10 \mathrm{~min} \text { before the skin } \\
\text { incision; Intravenous, } 10 \mathrm{mg} / \mathrm{kg}, 3,6,12 \text {, and } 24 \mathrm{~h} \text { after } \\
\text { operation; Topical, } 1 \mathrm{~g} \text {, intraoperatively }\end{array}$ \\
\hline Ma 2017 & $100 \mathrm{~mm} \mathrm{Hg}$ above systolic pressure & NA & $\begin{array}{l}\text { Intravenously, } 20 \mathrm{mg} / \mathrm{kg} \text {, anesthesia induction; Topical, } \\
1 \mathrm{~g} \text {, intraoperatively; Intravenous, } 10 \mathrm{mg} / \mathrm{kg}, 3,6,12 \text {, } \\
24 \mathrm{~h} \text { after anesthesia induction }\end{array}$ \\
\hline Palanne $2020^{1}$ & $250 \mathrm{mmHg}$ & After bandages applied & $\begin{array}{l}\text { Intravenously, } 1 \mathrm{~g}, 5 \mathrm{~min} \text { before surgery; Topical, } 1 \mathrm{~g} \text {, } \\
\text { intraoperatively; } 1 \mathrm{~g}, 3 \mathrm{~h}, 6 \mathrm{~h} \text { after surgery }\end{array}$ \\
\hline Palanne $2020^{2}$ & $250 \mathrm{mmHg}$ & After bandages applied & $\begin{array}{l}\text { Intravenously, } 1 \mathrm{~g}, 5 \mathrm{~min} \text { before surgery; Topical, } 1 \mathrm{~g} \text {, } \\
\text { intraoperatively; } 1 \mathrm{~g}, 3 \mathrm{~h}, 6 \mathrm{~h} \text { after surgery }\end{array}$ \\
\hline Wang 2017 & $300 \mathrm{mmHg}$ & After bandages applied & $\begin{array}{l}\text { Intravenously, } 1 \text { g, } 15 \text { min before surgery; Topical, } 1 \text { g, } \\
\text { intraoperatively }\end{array}$ \\
\hline Wang 2019 & NA & After bandages applied & $\begin{array}{l}\text { Intravenously, } 1 \mathrm{~g}, 15 \text { min before surgery; Topical, } 1 \mathrm{~g} \text {, } \\
\text { intraoperatively; Intravenously, } 1 \mathrm{~g}, 3 \mathrm{~h} \text { after surgery }\end{array}$ \\
\hline Xie 2017 & $100 \mathrm{~mm} \mathrm{Hg}$ above systolic pressure & After bandages applied & $\begin{array}{l}\text { Intravenously, } 20 \mathrm{mg} / \mathrm{kg} \text {, } 10 \mathrm{~min} \text { before surgery; Topical, } \\
60 \mathrm{ml} \text {, intraoperatively }\end{array}$ \\
\hline Xue 2018 & $100 \mathrm{~mm} \mathrm{Hg}$ above systolic pressure & After fascia layer closed & Intravenously, 1 kg, 30 min before surgery \\
\hline Yu 2017 & $300 \mathrm{mmHg}$ & After bandages applied & Topical, $1 \mathrm{~g}$, intraoperatively \\
\hline Zak 2021 & NA & NA & $\begin{array}{l}\text { Intravenously, two dose of } 1 \mathrm{~g} \text {, before surgery and dur- } \\
\text { ing wound closure }\end{array}$ \\
\hline Zeng 2021 & $100 \mathrm{mmHg}$ above systolic blood pressure & After bandages applied & Intravenously, 1 kg, before surgery \\
\hline Zhou 2017 & NA & NA & $\begin{array}{l}\text { Intravenously, } 1 \mathrm{~g} \text {, at the initiation of the surgery and } \\
\text { just before closure }\end{array}$ \\
\hline
\end{tabular}

The detailed information of tourniquet pressure, tourniquet inflation time, tourniquet realizing time of two groups

Table 4 The detailed information of TXA and Thromboprophylaxis drugs

\begin{tabular}{|c|c|c|}
\hline Author/year & TXA administration & Thromboprophylaxis drugs \\
\hline Alexandersson 2018 & Intravenously, $1 \mathrm{~g}, 10$ min before surgery & Low-molecular weight heparin \\
\hline Concina 2019 & Intravenously, $15 \mathrm{mg} / \mathrm{kg}, 20 \mathrm{~min}$ before surgery and after $4 \mathrm{~h}$ & Enoxaparine $4000 \mathrm{IU}$ \\
\hline Ejaz 2014 & Orally, $1 \mathrm{~g}$, before surgery; orally, $0.5 \mathrm{~g} 3 \mathrm{~h}$ after surgery & Rivaroxaban (10 mg/day) \\
\hline Huang 2017 & $\begin{array}{l}\text { Intravenously, } 20 \mathrm{mg} / \mathrm{kg}, 5 \text { to } 10 \text { min before the skin incision; Intravenous, } 10 \mathrm{mg} / \mathrm{kg}, 3,6,12 \text {, and } \\
24 \mathrm{~h} \text { after operation; Topical, } 1 \mathrm{~g} \text {, intraoperatively }\end{array}$ & Enoxaparine $4000 \mathrm{IU}$ \\
\hline Ma 2017 & $\begin{array}{l}\text { Intravenously, } 20 \mathrm{mg} / \mathrm{kg} \text {, anesthesia induction; Topical, } 1 \mathrm{~g} \text {, intraoperatively; Intravenous, } 10 \mathrm{mg} \text { / } \\
\mathrm{kg}, 3,6,12,24 \mathrm{~h} \text { after anesthesia induction }\end{array}$ & Enoxaparine $4000 \mathrm{IU}$ \\
\hline Palanne $2020^{1}$ & Intravenously, 1 g, 5 min before surgery; Topical, $1 \mathrm{~g}$, intraoperatively; $1 \mathrm{~g}, 3 \mathrm{~h}, 6 \mathrm{~h}$ after surgery & NA \\
\hline Palanne $2020^{2}$ & Intravenously, 1 g, 5 min before surgery; Topical, 1 g, intraoperatively; $1 \mathrm{~g}, 3 \mathrm{~h}, 6 \mathrm{~h}$ after surgery & NA \\
\hline Wang 2017 & Intravenously, $1 \mathrm{~g}$, 15 min before surgery; Topical, 1 g, intraoperatively & Rivaroxaban (10 mg/day) \\
\hline Wang 2019 & $\begin{array}{l}\text { Intravenously, } 1 \text { g, } 15 \text { min before surgery; Topical, } 1 \text { g, intraoperatively; Intravenously, } 1 \mathrm{~g}, 3 \mathrm{~h} \\
\text { after surgery }\end{array}$ & Enoxaparine $4000 \mathrm{IU}$ \\
\hline Xie 2017 & Intravenously, 20 mg/kg, 10 min before surgery; Topical, 60 ml, intraoperatively & Enoxaparine $4000 \mathrm{IU}$ \\
\hline Xue 2018 & Intravenously, 1 kg, 30 min before surgery & Rivaroxaban (10 mg/day) \\
\hline Yu 2017 & Topical, 1 g, intraoperatively & Rivaroxaban (10 mg/day) \\
\hline Zak 2021 & Intravenously, two dose of $1 \mathrm{~g}$, before surgery and during wound closure & NA \\
\hline Zeng 2021 & Intravenously, 1 kg, before surgery & Rivaroxaban (10 mg/day) \\
\hline Zhou 2017 & Intravenously, $1 \mathrm{~g}$, at the initiation of the surgery and just before closure & Rivaroxaban (10 mg/day) \\
\hline
\end{tabular}

The detailed information of TXA and thromboprophylaxis drugs of two groups. h, hour; min, minute; IU, international unit; kg, kilogram; g, gram; mg, milligram; ml, milliliter 


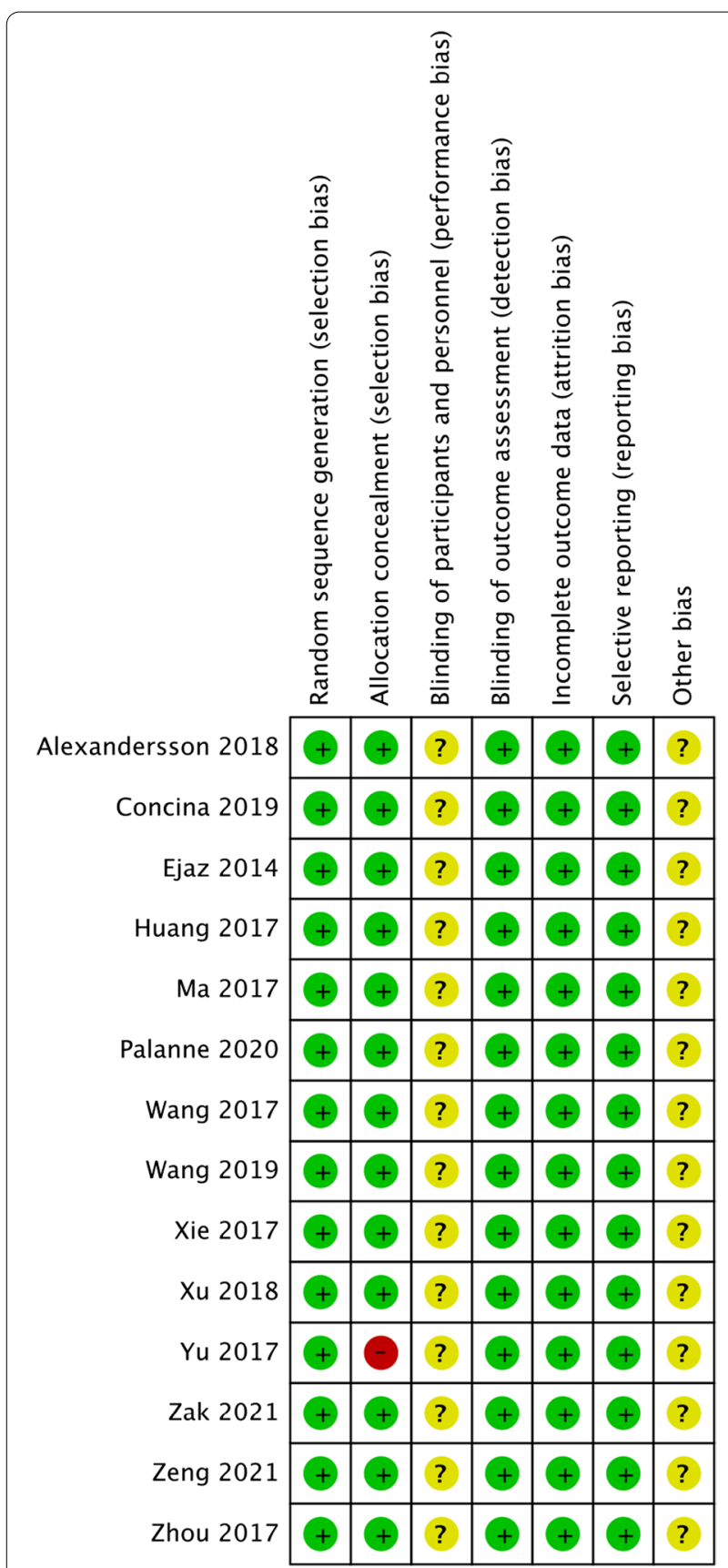

Fig. 2 Risk of bias summary: + no bias; - : bias; ?: bias unknown. Fourteen studies adequately described the correct randomization. Thirteen studies demonstrated sufficient allocation concealment. Four studies described the blinding of participants and personnel. No studies described the blinding of outcome assessment. All thirteen articles retained complete outcome data and avoided selective reporting. We rated as unclear risk of other bias because we can't ignore other potential dangers of biases. As a result, there is low or moderate risk of bias in most of the articles reviewed

\section{Complications}

Five RCTs reported intramuscular venous thrombosis. Six RCTs reported deep venous thrombosis, five RCTs reported superficial infection, and four RCTs reported deep wound infection. Four RCTs reported delayed wound healing. We detected no significantly difference in terms of intramuscular venous thrombosis $(\mathrm{RD}=0.01$, 95\% CI $-0.04,0.05], P=0.81$; Fig. 14), deep venous thrombosis ( $\mathrm{RD}=0.03,95 \% \mathrm{CI}-0.00,0.05], P=0.10$; Fig. 14), superficial infection $(\mathrm{RD}=0.01,95 \% \mathrm{CI}-0.02$, $0.05], P=0.45$; Fig. 14), deep wound infection $(\mathrm{RD}=0.01$, 95\% CI $-0.02,0.04], P=0.64$; Fig. 14), delayed wound healing ( $\mathrm{RD}=0.01,95 \% \mathrm{CI}-0.03,0.04], P=0.65$; Fig. 14 ) between two groups.

\section{Discussion}

Our study is the first meta-analysis to identify relevant randomized controlled trials involving TXA plus a tourniquet and use of TXA plus no tourniquet during TKA. This meta-analysis of 15 RCTs that evaluated a total of 1720 TKAs shows that TXA plus tourniquet group can decrease intraoperative blood loss and surgery duration, however increase hidden blood loss and decrease the knee ROM. Our findings suggested that there were no significant differences in terms of total blood loss, decrease in hemoglobin, transfusion rate, drainage volume, VAS, HSS, knee circumference, knee ROM, LOH, and complications between the two groups.

The result showing that the use of a tourniquet plus TXA effectively reduced intraoperative blood loss was consistent with the outcome of previous meta-analysis [35-37]. However, we found the TXA-T group has more hidden blood loss. An explanation for these conflicting results of IBL and HBL indicates that hidden blood loss plays a key role. Tourniquet release can result in ongoing bleeding from cut cancellous bone [38], blood extravasated into the knee joint and adjacent soft tissues [39], or blood loss from hemolysis [40] because of tourniquetinduced ischemia [41, 42]. Furthermore, there are no differences in drainage volume and total blood loss between the two groups, which is inconsistent with the previous meta-analysis. At an earlier meta-analysis [13, 37, 43], they found total blood loss to be significantly lower with a tourniquet. We think the reason for the difference between our study and previous meta- analysis [13, 37, 43 ] is the TXA used in all RCT studies included in our meta-analysis.

Hemoglobin level and transfusion rate have been recognized as the most objective indicators of actual blood loss. The decrease in hemoglobin and transfusion rate was similar in the TXA-NT group compared with the TXA-T group in our study. Blood transfusion 


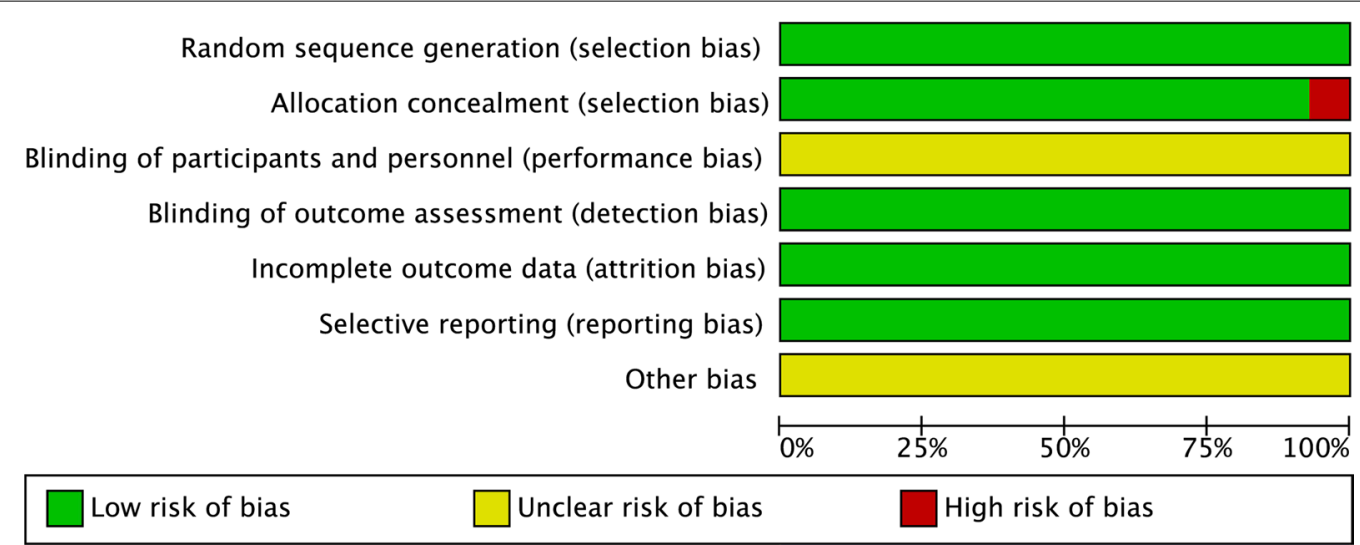

Fig. 3 Risk of bias graph. The overall quality of the included studies was considered adequate

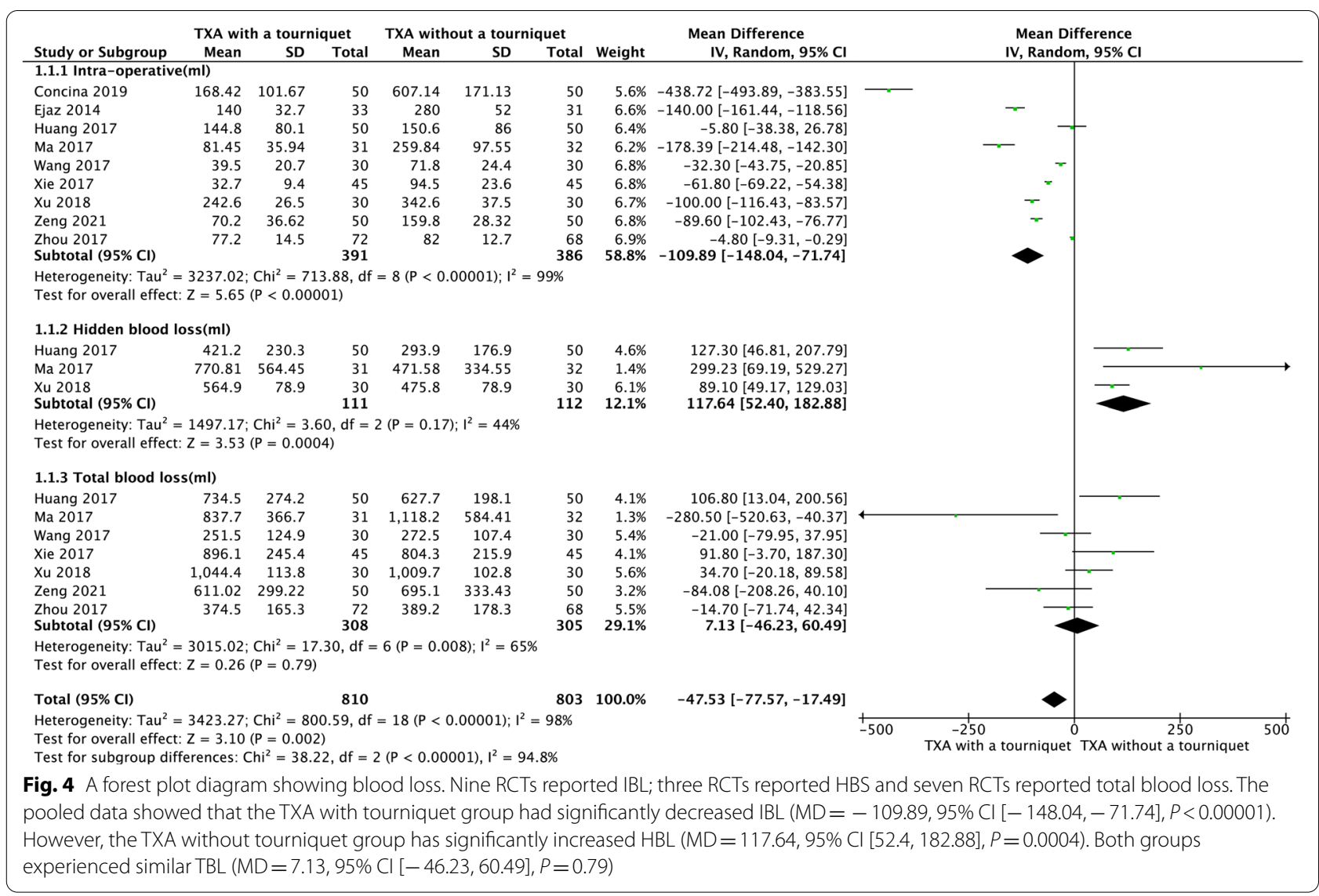

is associated with adverse effects, including hemolytic reactions, infections, morbidity, immunologically mediated diseases, and cost [44]. The result of similar transfusion rate in both groups is consistent with Cai's recent meta-analysis [45]. They found no significant difference between the tourniquet group and the non-tourniquet group.
A tourniquet will provide surgeons with a bloodless surgery field to facilitate the clear identification of anatomical structures with less electrocoagulation and wound irrigation during surgery, which might help shorten the operation time. Our result showed tourniquet with TXA use reduced surgery duration, which was consistent with previous studies [2, 35, 38]. So a 


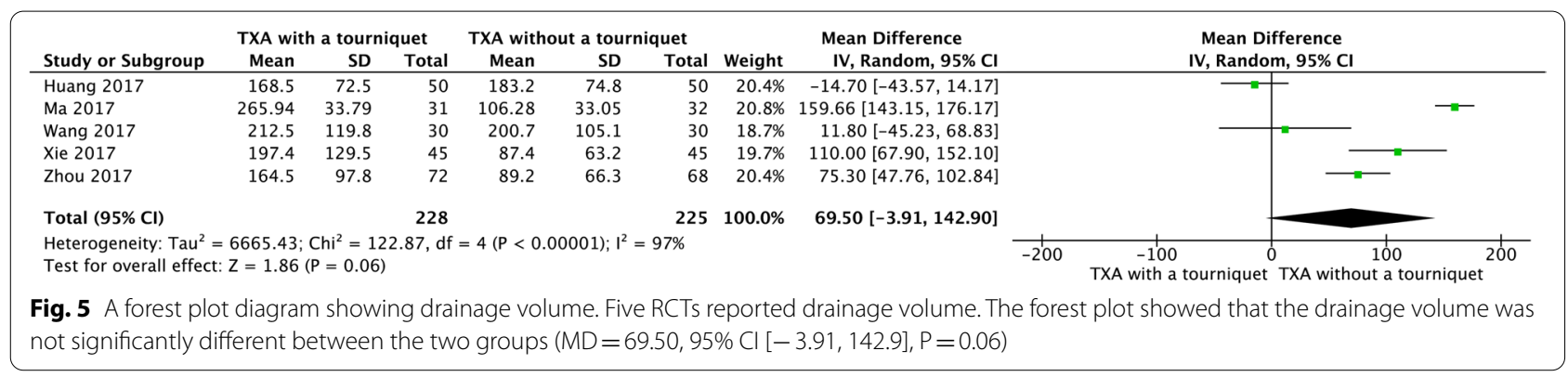

\begin{tabular}{|c|c|c|c|c|c|c|c|c|c|c|c|}
\hline \multirow[b]{2}{*}{ Study or Subgroup } & \multicolumn{3}{|c|}{ Experimental } & \multicolumn{3}{|c|}{ Control } & \multirow[b]{2}{*}{ Weight } & \multirow{2}{*}{$\begin{array}{l}\text { Mean Difference } \\
\text { IV, Random, } 95 \% \mathrm{Cl}\end{array}$} & \multirow{2}{*}{\multicolumn{3}{|c|}{$\begin{array}{l}\text { Mean Difference } \\
\text { IV, Random, } 95 \% \mathrm{Cl}\end{array}$}} \\
\hline & Mean & SD & Total & Mean & SD & Total & & & & & \\
\hline Ma 2017 & 26.42 & 9.62 & 31 & 20.91 & 6.89 & 32 & $24.0 \%$ & $5.51[1.37,9.65]$ & \multirow{4}{*}{-} & \multicolumn{2}{|l|}{--} \\
\hline Palanne 2020(1) & 25 & 7.7 & 101 & 31 & 8.6 & 99 & $26.0 \%$ & $-6.00[-8.26,-3.74]$ & & & \\
\hline Palanne 2020(2) & 25 & 7.9 & 99 & 29 & 8.1 & 96 & $26.0 \%$ & $-4.00[-6.25,-1.75]$ & & \multirow[b]{2}{*}{--} & \\
\hline Xie 2017 & 26.4 & 11.2 & 45 & 18.5 & 8.3 & 45 & $24.0 \%$ & $7.90[3.83,11.97]$ & & & \\
\hline \multicolumn{3}{|l|}{ Total $(95 \% \mathrm{Cl})$} & 276 & & & 272 & $100.0 \%$ & $0.62[-5.44,6.68]$ & \multicolumn{2}{|c|}{ 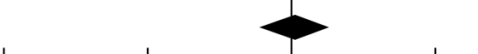 } & \\
\hline \multicolumn{8}{|c|}{$\begin{array}{l}\text { Heterogeneity: } \text { Tau }^{2}=35.41 ; \mathrm{Chi}^{2}=50.20, \mathrm{df}=3(\mathrm{P}<0.00001) ; \mathrm{I}^{2}=94 \% \\
\text { Test for overall effect: } Z=0.20(P=0.84)\end{array}$} & $+_{-50}^{+}$ & $\begin{array}{l}1 \\
0 \\
\text { Favours [experimental] }\end{array}$ & $\frac{1}{25}$ & 50 \\
\hline \multicolumn{12}{|c|}{$\begin{array}{l}\text { Fig. } 6 \text { A forest plot diagram showing decrease in hemoglobin. Four RCTs reported a decrease in hemoglobin. The pooled data revealed that the } \\
\text { reduction in hemoglobin was not significantly different between the two groups ( } M D=7.90,95 \% \mathrm{Cl}[-5.44,6.68], P=0.84)\end{array}$} \\
\hline
\end{tabular}

\begin{tabular}{|c|c|c|c|c|c|c|c|}
\hline \multirow[b]{2}{*}{ Study or Subgroup } & \multicolumn{2}{|c|}{ TXA with tourniquet } & \multicolumn{2}{|c|}{ TXA without tourniquet } & \multirow[b]{2}{*}{ Weight } & \multirow{2}{*}{$\begin{array}{l}\text { Risk Difference } \\
\text { M-H, Fixed, } 95 \% \mathrm{Cl}\end{array}$} & \multirow{2}{*}{$\begin{array}{l}\text { Risk Difference } \\
\mathrm{M}-\mathrm{H}, \text { Fixed, } 95 \% \mathrm{CI}\end{array}$} \\
\hline & Events & Total & Events & Total & & & \\
\hline Concina 2019 & 4 & 50 & 1 & 50 & $10.8 \%$ & $0.06[-0.02,0.14]$ & $\begin{array}{ll} & \\
\end{array}$ \\
\hline Huang 2017 & 0 & 50 & 0 & 50 & $10.8 \%$ & $0.00[-0.04,0.04]$ & \multirow{2}{*}{ 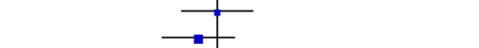 } \\
\hline Palanne 2020(1) & 1 & 101 & 3 & 99 & $21.6 \%$ & $-0.02[-0.06,0.02]$ & \\
\hline Palanne 2020(2) & 1 & 99 & 1 & 96 & $21.1 \%$ & $-0.00[-0.03,0.03]$ & \\
\hline Xie 2017 & 1 & 45 & 0 & 45 & $9.7 \%$ & $0.02[-0.04,0.08]$ & \multirow{2}{*}{$\longrightarrow$} \\
\hline Zeng 2021 & 0 & 50 & 0 & 50 & $10.8 \%$ & $0.00[-0.04,0.04]$ & \\
\hline Zhou 2017 & 8 & 72 & 3 & 68 & $15.1 \%$ & $0.07[-0.02,0.15]$ & $\longrightarrow$ \\
\hline Total $(95 \% \mathrm{Cl})$ & \multicolumn{2}{|r|}{467} & & 458 & $100.0 \%$ & $0.01[-0.01,0.04]$ & 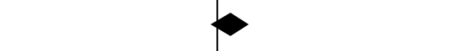 \\
\hline \multirow{2}{*}{\multicolumn{7}{|c|}{$\begin{array}{l}\text { Heterogeneity: } \mathrm{Chi}^{2}=7.74, \mathrm{df}=6(\mathrm{P}=0.26) ; \mathrm{I}^{2}=22 \% \\
\text { Test for overall effect: } Z=1.34(\mathrm{P}=0.18)\end{array}$}} & \\
\hline & & & & & & & 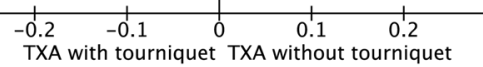 \\
\hline
\end{tabular}

\begin{tabular}{|c|c|c|c|c|c|c|c|c|c|c|}
\hline Study or Subgroup & \multicolumn{3}{|c|}{ Experimental } & \multicolumn{3}{|c|}{ Control } & \multicolumn{2}{|r|}{ Mean Difference } & \multicolumn{2}{|c|}{$\begin{array}{l}\text { Mean Difference } \\
\text { IV, Fixed, } 95 \% \mathrm{Cl}\end{array}$} \\
\hline Ejaz 2014 & 70 & 1 & 33 & 71 & 0.75 & 31 & $88.3 \%$ & $-1.00[-1.43,-0.57]$ & \multicolumn{2}{|l|}{ 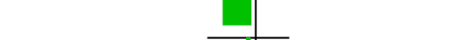 } \\
\hline Huang 2017 & 51.8 & 4.6 & 50 & 52.2 & 6.2 & 50 & $3.6 \%$ & $-0.40[-2.54,1.74]$ & $\rightarrow$ & - \\
\hline Ma 2017 & 57.81 & 7.94 & 31 & 62.28 & 7.15 & 32 & $1.2 \%$ & $-4.47[-8.20,-0.74]$ & \multirow{2}{*}{-} & \\
\hline Wang 2017 & 25.3 & 2.7 & 30 & 26.3 & 3.7 & 30 & $6.1 \%$ & $-1.00[-2.64,0.64]$ & & \\
\hline Zhou 2017 & 77.2 & 14.5 & 72 & 82 & 12.7 & 68 & $0.8 \%$ & $-4.80[-9.31,-0.29]$ & & \\
\hline Total $(95 \% \mathrm{Cl})$ & & & 216 & & & 211 & $100.0 \%$ & $-1.05[-1.46,-0.64]$ & \multicolumn{2}{|l|}{$\checkmark$} \\
\hline \multicolumn{6}{|c|}{$\begin{array}{l}\text { Heterogeneity: } \mathrm{Chi}^{2}=6.29, \mathrm{df}=4(\mathrm{P}=0.18) ; \mathrm{I}^{2}=36 \% \\
\text { Test for overall effect: } \mathrm{Z}=5.08(\mathrm{P}<0.00001)\end{array}$} & & & & \multicolumn{2}{|c|}{ 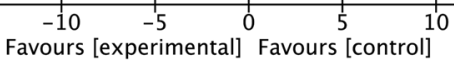 } \\
\hline
\end{tabular}

reduction of course of surgery is a potential benefit of tourniquet use with TXA in TKA.

Pain relief in the early postoperative period after TKA is crucial in facilitating early recovery. Whether the use of tourniquets will increase postoperative pain remains controversial. Theoretically, tourniquet use may increase thigh pain and swell due to lower limb blood flow occlusion and ischemia-reperfusion injury. 


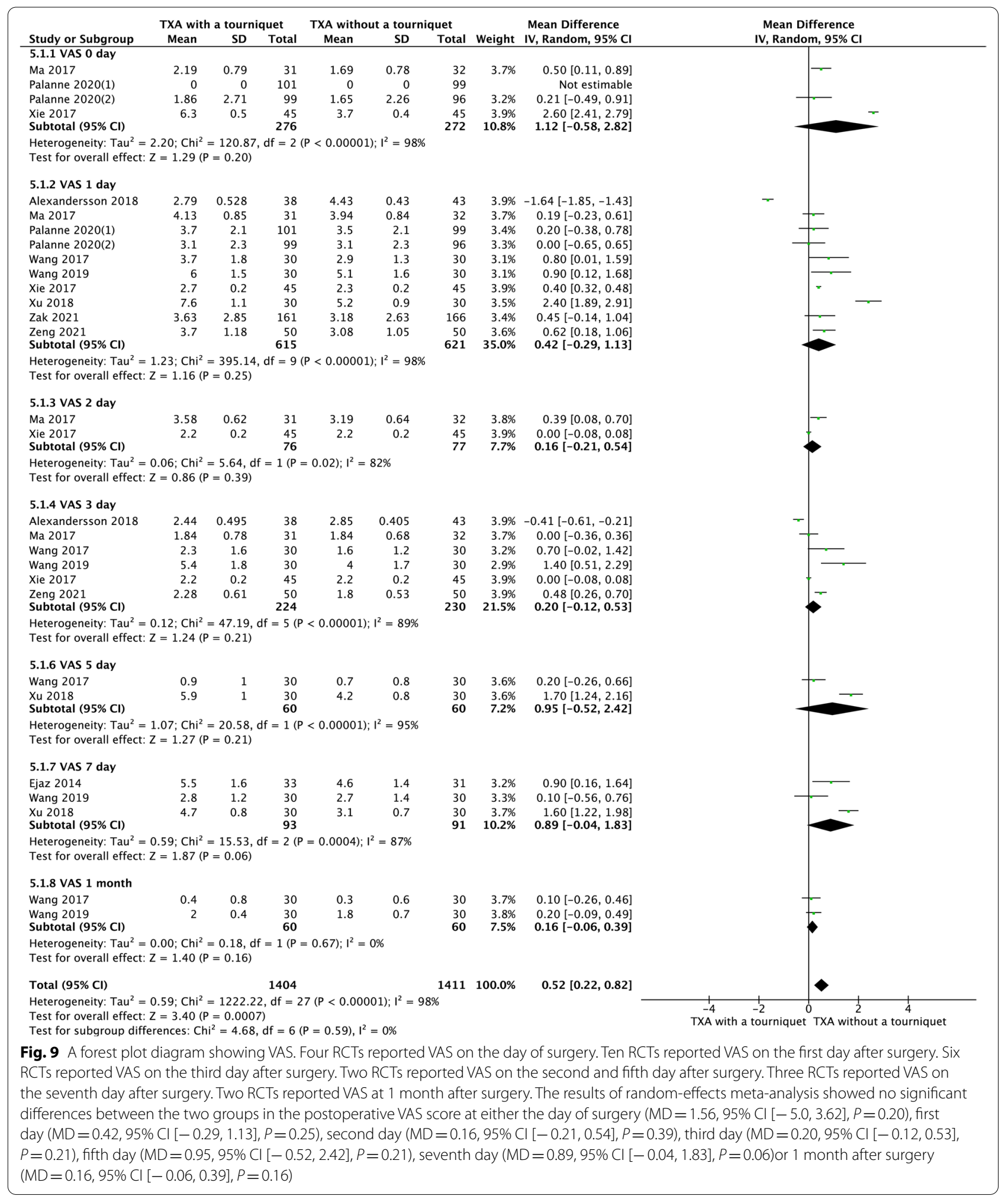

Our study identified no difference in pain intensity at either the day of surgery, first day, second day, the third day, fifth day, the seventh day, or 1 month after surgery.
Although tourniquet pressure, time, and time of postoperative pain evaluation were variable across studies, we found that these factors of all included RCTs were 


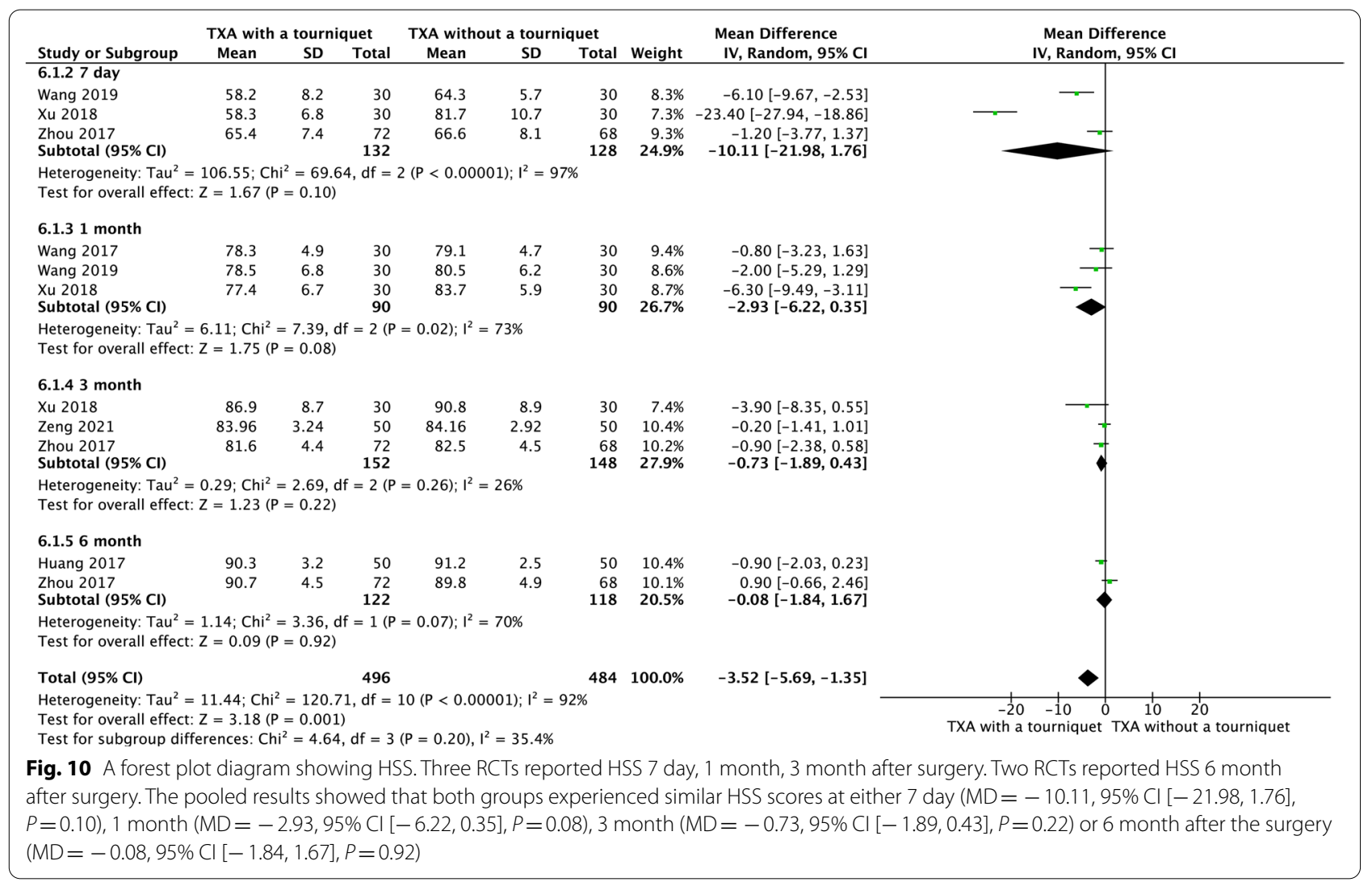

\begin{tabular}{|c|c|c|c|c|c|c|c|c|c|}
\hline \multirow[b]{2}{*}{ Study or Subgroup } & \multicolumn{3}{|c|}{ TXA with a tourniquet } & \multicolumn{3}{|c|}{ TXA without a tourniquet } & \multirow[b]{2}{*}{ Weight } & \multirow{2}{*}{$\begin{array}{l}\text { Mean Difference } \\
\text { IV, Random, } 95 \% \mathrm{CI}\end{array}$} & \multirow{2}{*}{$\begin{array}{l}\text { Mean Difference } \\
\text { IV, Random, } 95 \% \mathrm{CI}\end{array}$} \\
\hline & Mean & SD & Total & Mean & SD & Total & & & \\
\hline \multicolumn{10}{|c|}{ 7.1.1 Knee circumference } \\
\hline Alexandersson 2018 & 46.8 & 1.2 & 38 & 46.3 & 0.25 & 43 & $50.4 \%$ & $0.50[0.11,0.89]$ & $\Gamma$ \\
\hline $\begin{array}{l}\text { Xu } 2018 \\
\text { Subtotal (95\% cl) }\end{array}$ & 52.6 & 3.7 & $\begin{array}{l}30 \\
68\end{array}$ & 41.3 & 3.6 & 30 & $49.6 \%$ & $11.30[9.45,13.15]$ & 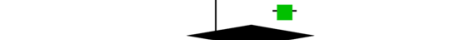 \\
\hline \multicolumn{10}{|c|}{$\begin{array}{l}\text { Heterogeneity: } \mathrm{Tau}^{2}=57.86 ; \mathrm{Chi}^{2}=125.73, \mathrm{df}=1(\mathrm{P}<0.00001) ; \mathrm{I}^{2}=99 \% \\
\text { Test for overall effect: } Z=1.09(\mathrm{P}=0.28)\end{array}$} \\
\hline \multirow{2}{*}{\multicolumn{9}{|c|}{$\begin{array}{l}\text { Total }(95 \% \mathrm{Cl}) \\
\text { Heterogeneity: } \text { Tau }^{2}=57.86 ; \mathrm{Chi}^{2}=125.73, \mathrm{df}=1 \\
\text { Test for overall effect: } Z=1.09(\mathrm{P}=0.28) \\
\text { Test for subgroup differences: Not applicable }\end{array}$}} & \\
\hline & & & & & & & & & 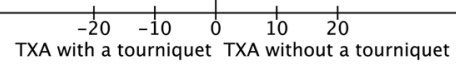 \\
\hline \multicolumn{10}{|c|}{$\begin{array}{l}\text { Fig. } 11 \text { A forest plot diagram showing knee circumference. Two RCTs reported knee circumference. We detected a similar knee circumference } \\
\text { between two groups }(\mathrm{MD}=5.86,95 \% \mathrm{Cl}-4.72,16.44], P=0.28)\end{array}$} \\
\hline
\end{tabular}

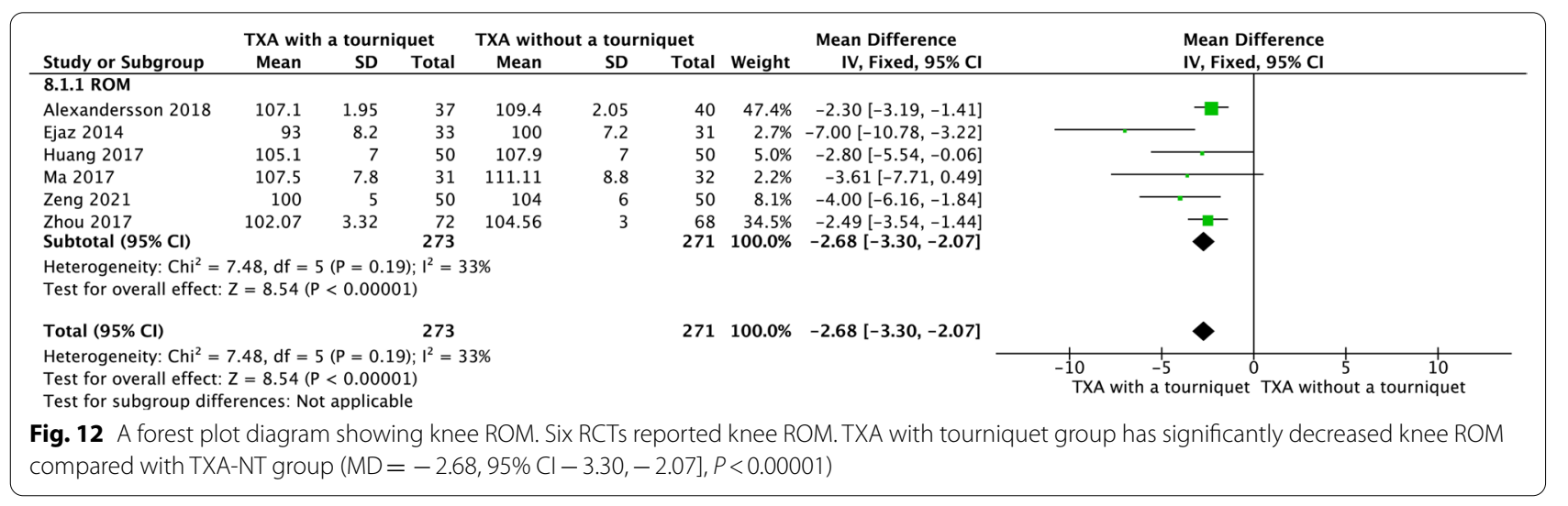




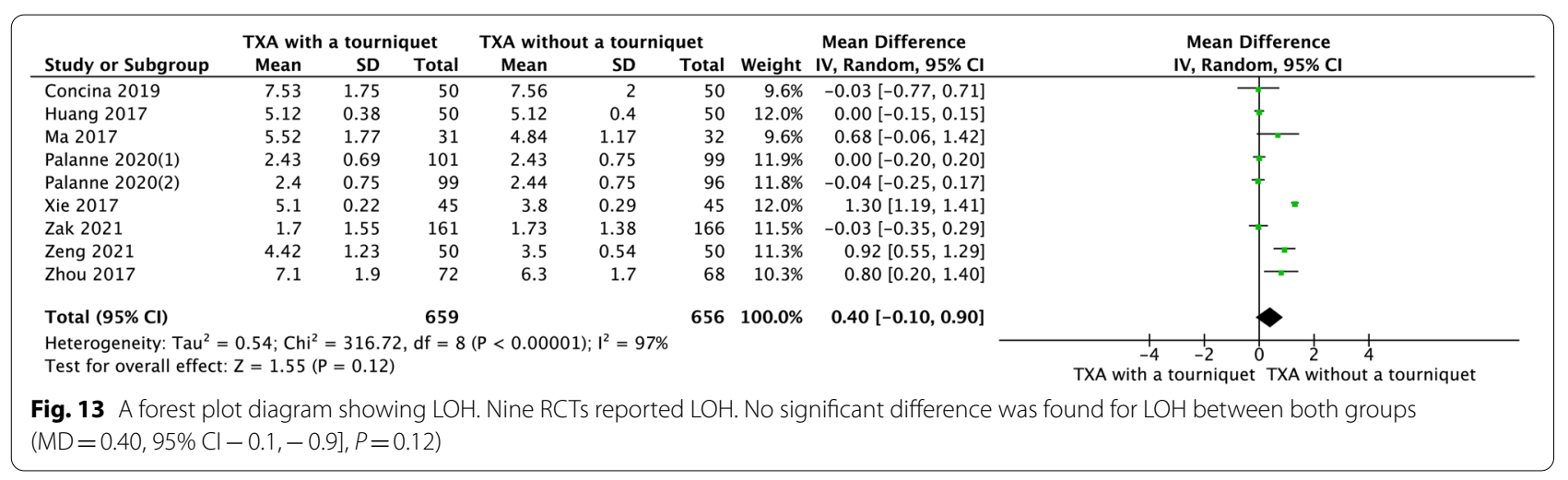

comparable between experimental and control groups, so endpoints like VAS, ROM, and LOS could still be properly assessed. We also have tried our best to evaluate VAS based on time points. Our results of VAS were inconsistent with previous studies. [25, 46, 47]. It may be related to the tourniquet pressure in our tourniquet group. In our study, lower or personalized tourniquet pressure was used in 5 of the 11 RCTs. Worland et al. [48] showed an essential correlation between higher tourniquet pressure and more thigh pain in the immediate postoperative period.

Knee flexion ROM is often used to evaluate short-term effectiveness. Besides, discharge from the hospital is dependent on the mobility of patients following TKA. We found significantly decreased knee ROM in TXA-T group compared with TXA-NT group, which is consistent with the previous systematic reviews $[35,49]$. We think the possible reasons are as follows: (1) using a tourniquet could injure the nerve and the skeletal muscle, even causing rhabdomyolysis [49]; (2) the tourniquet might cause reperfusion injury, which might cause a degree of muscle fibrosis; (3) there was some delay in the nerve conduction and electromyography changes of the extensor apparatus when using tourniquet [49]. No significant difference was also found in terms of knee circumference between the two groups. These findings seem logical, given that we found no significant difference in terms of VAS.

The analysis of the postoperative HSS at either 7 days, 1-month, 3 months or 6 months after the surgery also did not reveal a difference. HSS might be affected by many factors such as pain, ROM, function, muscle force, and flexion deformity. Moreover, the effect of a tourniquet application plus TXA on HSS needs to be further confirmed by more high-quality studies.

As for complications, we observed no significant difference in terms of IMVT, DVT, superficial infection, deep wound infection, delayed wound healing between the two groups. Although TXA use in TKA didn't increase thromboembolic events [50-53], perhaps one of the more significant clinical concerns regarding tourniquet use plus TXA is its association with thromboembolism. No significant difference was found between groups regarding the rate of intramuscular venous thrombosis and deep venous thrombosis in our study. Several studies have investigated the incidence of venous thrombosis with the use of the tourniquet [3, $13,14,36,54]$. However, the evidence is mixed because of heterogeneous study groups and designs, making it difficult to compare. Nonetheless, we cannot underscore the importance of chemoprophylaxis following TKA regardless of tourniquet use. DVT was detected in $81 \%$ of patients when all the patents only received mechanical compression but no chemoprophylaxis following TKA of tourniquet use [55].

The current meta-analysis has several limitations: First, there is a high heterogeneity of blood loss caused by the different methods for measuring blood loss, separate application of a tourniquet, different operative techniques, and different perioperative management as the drain and anticoagulant therapy. The reliability of results may be influenced by this heterogeneity. Second, the studies' comparability was complicated through the different measurement methods and follow-up examination time points; however, we have tried our best to evaluate results based on time points. Third, the tourniquet time, the time for loosening the tourniquet, and the cuff pressure used were also not uniform. Fourth, there are no worldwide uniform guidelines for performing total knee arthroplasty. Different surgical techniques (such as the selection of approach, anesthesia methods, patellar resurfacing, and type of prosthesis) were used in the individual studies. Fifth, the tourniquet may have impact on patella tracking; however, there were too few studies comparing patella tracking between two groups, so we did not have further research on the patella tracking in our study. 


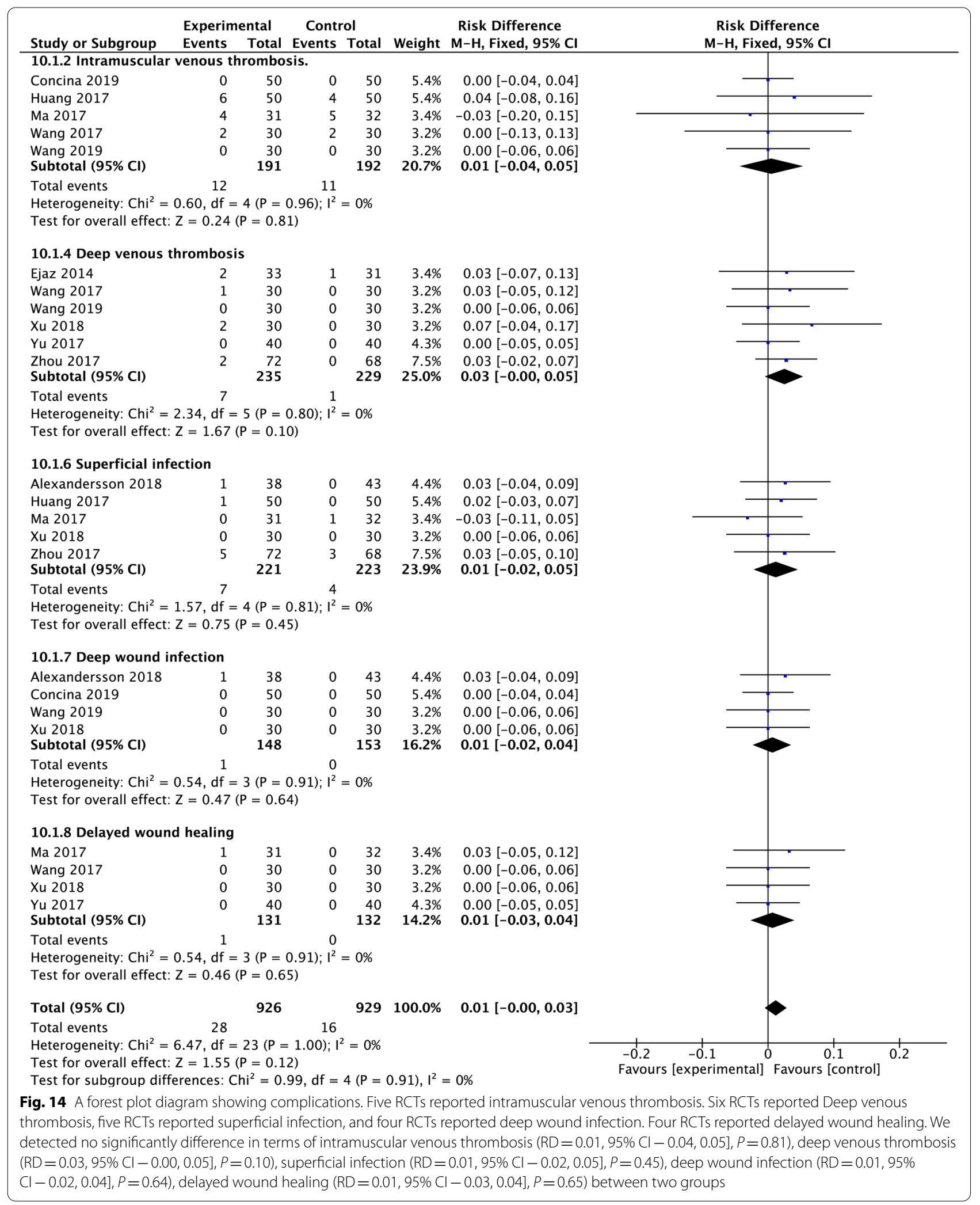




\section{Conclusion}

No big differences could be found by using or not tourniquet with TXA. Some benefits are related to operation time and less intra-operative blood loss by using tourniquet and TXA; however, using the tourniquet and TXA was also related to more hidden blood loss and less knee range of motion. These are obvious conclusions that are confirmed after this meta-analysis. Given our meta-analysis' relevant possible biases, we required more adequately powered and better-designed RCT studies with long-term follow-up to reach a firmer conclusion.

\begin{abstract}
Abbreviations
Cis: Confidence intervals; RCTs: Randomized controlled trials; RR: Risk ratio; OR: Odds ratio; VMD: Weighted mean difference; TXA: Tranexamic acid; TKA: Total knee arthroplasty; OA: Osteoarthritis; RA: Rheumatoid arthritis; BMI: Body mass index; VAS: Visual Analogue Scale; HSS: Hospital for Special Surgery; ROM: Range of motion; IBL: Intra-operative blood loss; HBL: Hidden blood loss; TBL: Total blood loss; LOH: Length of stay; IMVT: Intramuscular venous thrombosis; DVT: Deep venous thrombosis.
\end{abstract}

\section{Acknowledgements}

None.

\section{Authors' contributions}

C.S. and Y.Z. contributed to the conceptualization. Q.M. and Y.T. contributed to the data curation. C.S. and Q.M. were involved in the formal analysis. X.Z. and C.S. were involved in the investigation. X.C. and Y.Z. contributed to the supervision. C.S. and Y.Z. contributed to the validation. C.S. and Y.Z. contributed to the visualization. C.S. was involved in the writing —original draft. All authors read and approved the final manuscript.

\section{Funding}

None.

\section{Availability of data and materials}

The datasets generated and analyzed during the current study are available from the corresponding author on reasonable request.

\section{Declarations}

\section{Consent for publication}

Not applicable.

\section{Ethics approval}

Ethical approval is not required, because this study is based on existed literature.

\section{Competing interests}

The authors declare that they have no competing interests.

\section{Author details}

${ }^{1}$ Department of Orthopedic, Beijing Tsinghua Changgung Hospital, School of Clinical Medicine, Tsinghua University, No. 168 Litang Road, Dongxiaokou Town, Changping District, Beijing 102218, China. ${ }^{2}$ Department of Clinical Epidemiology and Biostatistics, Beijing Tsinghua Changgung Hospital, Schoo of Clinical Medicine, Tsinghua University, No.1 68 Litang Road, Dongxiaokou Town, Changping District, Beijing 102218, China. ${ }^{3}$ Department of Nursing, Beijing Tsinghua Changgung Hospital, School of Clinical Medicine,, Tsinghua University, No. 168 Litang Road, Dongxiaokou Town, Changping District, Beijing 102218, China. ${ }^{4}$ Department of Orthopaedic Surgery, The First Medical Centre, Chinese PLA General Hospital, No. 28 Fuxing Road, Beijing 100853, China.
Received: 11 November 2021 Accepted: 30 December 2021

Published online: 15 January 2022

\section{References}

1. Huang ZY, Pei FX, Ma J, Yang J, Zhou ZK, Kang PD, Shen B. Comparison of three different tourniquet application strategies for minimally invasive total knee arthroplasty: a prospective non-randomized clinical trial. Arch Orthop Trauma Surg. 2014;134(4):561-70.

2. Zhou K, Ling T, Wang H, Zhou Z, Shen B, Yang J, Kang P, Pei F. Influence of tourniquet use in primary total knee arthroplasty with drainage: a prospective randomised controlled trial. J Orthop Surg Res. 2017;12(1):172.

3. Zhang $P$, Liang $Y$, He J, Fang $Y$, Chen P, Wang J. Timing of tourniquet release in total knee arthroplasty: a meta-analysis. Medicine (Baltimore). 2017;96(17):e6786.

4. Pfitzner T, von Roth P, Voerkelius N, Mayr H, Perka C, Hube R. Influence of the tourniquet on tibial cement mantle thickness in primary total knee arthroplasty. Knee Surg Sports Traumatol Arthrosc. 2016;24(1):96-101.

5. Young SW, Zhang M, Moore GA, Pitto RP, Clarke HD, Spangehl MJ. The John N. Insall Award: higher tissue concentrations of vancomycin achieved with intraosseous regional prophylaxis in revision TKA: a randomized controlled trial. Clin Orthop Relat Res. 2018;476(1):66-74.

6. Olivecrona C, Blomfeldt R, Ponzer S, Stanford BR, Nilsson BY. Tourniquet cuff pressure and nerve injury in knee arthroplasty in a bloodless field: a neurophysiological study. Acta Orthop. 2013;84(2):159-64.

7. Tsubosaka M, Ishida K, Sasaki H, Shibanuma N, Kuroda R, Matsumoto T. Effects of suture and tourniquet on intraoperative kinematics in navigated total knee arthroplasty. J Arthroplasty. 2017;32(6):1824-8.

8. Tai TW, Chang CW, Lai KA, Lin CJ, Yang CY. Effects of tourniquet use on blood loss and soft-tissue damage in total knee arthroplasty: a randomized controlled trial. J Bone Jt Surg Am. 2012;94(24):2209-15.

9. Tai TW, Lin CJ, Jou IM, Chang CW, Lai KA, Yang CY. Tourniquet use in total knee arthroplasty: a meta-analysis. Knee Surg Sports Traumatol Arthrosc. 2011;19(7):1121-30

10. Horlocker TT, Hebl JR, Gali B, Jankowski CJ, Burkle CM, Berry DJ, Zepeda FA, Stevens SR, Schroeder DR. Anesthetic, patient, and surgical risk factors for neurologic complications after prolonged total tourniquet time during total knee arthroplasty. Anesth Analg. 2006;102(3):950-5.

11. Silver R, de la Garza J, Rang M, Koreska J. Limb swelling after release of a tourniquet. Clin Orthop Relat Res. 1986;206:86-9.

12. Dennis DA, Kittelson AJ, Yang CC, Miner TM, Kim RH, Stevens-Lapsley JE. Does tourniquet use in TKA affect recovery of lower extremity strength and function? A randomized trial. Clin Orthop Relat Res. 2016;474(1):69-77.

13. Jiang FZ, Zhong HM, Hong YC, Zhao GF. Use of a tourniquet in total knee arthroplasty: a systematic review and meta-analysis of randomized controlled trials. J Orthop Sci. 2015;20(1):110-23.

14. Mori N, Kimura S, Onodera T, Iwasaki N, Nakagawa I, Masuda T. Use of a pneumatic tourniquet in total knee arthroplasty increases the risk of distal deep vein thrombosis: a prospective, randomized study. Knee. 2016;23(5):887-9.

15. Clarke MT, Longstaff L, Edwards D, Rushton N. Tourniquet-induced wound hypoxia after total knee replacement. J Bone Jt Surg Br. 2001;83(1):40-4.

16. Heller S, Chen A, Restrepo C, Albert E, Hozack WJ. Tourniquet release prior to dressing application reduces blistering following total knee arthroplasty. J Arthroplasty. 2015;30(7):1207-10.

17. Rathod P, Deshmukh A, Robinson J, Greiz M, Ranawat A, Rodriguez J. Does tourniquet time in primary total knee arthroplasty influence clinical recovery? J Knee Surg. 2015;28(4):335-42.

18. Barr L, lyer US, Sardesai A, Chitnavis J. Tourniquet failure during total knee replacement due to arterial calcification: case report and review of the literature. J Perioper Pract. 2010;20(2):55-8.

19. Xie J, Ma J, Yao H, Yue C, Pei F. Multiple boluses of intravenous tranexamic acid to reduce hidden blood loss after primary total knee arthroplasty without tourniquet: a randomized clinical trial. J Arthroplasty. 2016;31(11):2458-64.

20. Huang Z, Ma J, Shen B, Pei F. Combination of intravenous and topical application of tranexamic acid in primary total knee arthroplasty: a prospective randomized controlled trial. J Arthroplasty. 2014;29(12):2342-6. 
21. Xie J, Ma J, Kang P, Zhou Z, Shen B, Yang J, Pei F. Does tranexamic acid alter the risk of thromboembolism following primary total knee arthroplasty with sequential earlier anticoagulation? A large, single center, prospective cohort study of consecutive cases. Thromb Res. 2015;136(2):234-8.

22. Palanne R, Rantasalo M, Vakkuri A, Madanat R, Olkkola KT, Lahtinen K, Reponen E, Linko R, Vahlberg T, Skants N. Effects of anaesthesia method and tourniquet use on recovery following total knee arthroplasty: a randomised controlled study. Br J Anaesth. 2020;125(5):762-72.

23. Alexandersson M, Wang EY, Eriksson S. A small difference in recovery between total knee arthroplasty with and without tourniquet use the first 3 months after surgery: a randomized controlled study. Knee Surg Sports Traumatol Arthrosc. 2019;27(4):1035-42.

24. Concina C, Crucil M, Fabbro S, Gherlinzoni F. Do tourniquet and drainage influence fast track in total knee arthroplasty? Our results on 151 cases. Acta Biomed. 2019;90(1-s):123-9.

25. Ejaz A, Laursen AC, Kappel A, Laursen MB, Jakobsen T, Rasmussen S, Nielsen PT. Faster recovery without the use of a tourniquet in total knee arthroplasty. Acta Orthop. 2014;85(4):422-6.

26. Huang Z, Xie X, Li L, Huang Q, Ma J, Shen B, Kraus VB, Pei F. Intravenous and topical tranexamic acid alone are superior to tourniquet use for primary total knee arthroplasty: a prospective, randomized controlled trial. J Bone Jt Surg Am. 2017;99(24):2053-61.

27. Ma J, Huang Z, Wang R, Xie J, Xu B, Pei F, Shen B. Comparison of efficacy and safety between with and without tourniquet under the program of expert consensus statement on anti-coagulation sequential antifibrinolysis during primary total knee arthroplasty. Chin J Bone Jt Surg. 2017;10(05):391-5.

28. Wang G, Rui C, Chen X, Guan J, Zhu J, Zhu Q. Effects of tourniquet use on enhanced recovery after surgery in total knee arthroplasty. Chin J Bone Jt Surg. 2017;10(01):27-32.

29. Wang X, Tan B, Yin J, Zhuo B. Restrictive use and non-use tourniquets during total knee arthroplasty. J Clin Rehabil Tissue Eng Res. 2019;23(28):4456-60.

30. Xie X, Yue C, Wang Z, Kang P, Zhou Z, Yang J, Shen B, Pei F. Total knee arthroplasty with or without tourniquet: a randomized controlled trial. Orthop J China. 2017;25(17):1572-6.

31. Xue $C$, Yao $Y, X i n g Y$. Analysis of the effect and safety of tourniquet used in total knee arthroplasty. Chin J Bone Jt Injury. 2018;33(06):626-8.

32. Yu Z, Wang T, Sun H, Yan X, Li S. To observe clinical effect of limited tourniquet usage in total knee arthroplasty. Orthop Biomech Mater Clin Study. 2017;14(01):24-8.

33. Zak SG, Yeroushalmi D, Long WJ, Meftah M, Schnaser E, Schwarzkopf R. Does the use of a tourniquet influence outcomes in total knee arthroplasty: a randomized controlled trial. J Arthroplasty. 2021;36(7):2492-6.

34. Zeng $Y, L i Y$, Si H, Wu Y, Li M. Y L, Shen B: Effects of tourniquet use on clinical outcomes and cement penetration in TKA when tranexamic acid administrated: a randomized controlled trial. BMC Musculoskelet Disord. 2021;22(1):126.

35. Zhang W, Li N, Chen S, Tan Y, Al-Aidaros M, Chen L. The effects of a tourniquet used in total knee arthroplasty: a meta-analysis. J Orthop Surg Res. 2014;9(1):13.

36. Tie K, Hu D, Qi Y, Wang H, Chen L. Effects of tourniquet release on total knee arthroplasty. Orthopedics. 2016;39(4):e642-650.

37. Alcelik I, Pollock RD, Sukeik M, Bettany-Saltikov J, Armstrong PM, Fismer P. A comparison of outcomes with and without a tourniquet in total knee arthroplasty: a systematic review and meta-analysis of randomized controlled trials. J Arthroplasty. 2012;27(3):331-40.

38. Zan P, Li G. Releasing of tourniquet before wound closure or not in total knee arthroplasty: a meta-analysis of randomized controlled trials. J Arthroplasty. 2015;30(7):1154.

39. Erskine JG, Fraser C, Simpson R, Protheroe K, Walker ID. Blood loss with knee joint replacement. J R Coll Surg Edinb. 1981;26(5):295-7.

40. Pattison E, Protheroe K, Pringle RM, Kennedy AC, Dick WC. Reduction in haemoglobin after knee joint surgery. Ann Rheum Dis. 1973;32(6):582-4.

41. Tetro AM, Rudan JF. The effects of a pneumatic tourniquet on blood loss in total knee arthroplasty. Can J Surg. 2001;44(1):33-8.

42. Li B, Wen Y, Wu H, Qian Q, Lin X, Zhao H. The effect of tourniquet use on hidden blood loss in total knee arthroplasty. Int Orthop. 2009;33(5):1263-8.
43. Li X, Yin L, Chen ZY, Zhu L, Wang HL, Chen W, Yang G, Zhang YZ. The effect of tourniquet use in total knee arthroplasty: grading the evidence through an updated meta-analysis of randomized, controlled trials. Eur J Orthop Surg Traumatol. 2014;24(6):973-86.

44. Lasocki S, Krauspe R, von Heymann C, Mezzacasa A, Chainey S, Spahn DR. PREPARE: the prevalence of perioperative anaemia and need for patient blood management in elective orthopaedic surgery: a multicentre, observational study. Eur J Anaesthesiol. 2015;32(3):160-7.

45. Cai DF, Fan $\mathrm{QH}$, Zhong $\mathrm{HH}$, Peng $\mathrm{S}$, Song $\mathrm{H}$. The effects of tourniquet use on blood loss in primary total knee arthroplasty for patients with osteoarthritis: a meta-analysis. J Orthop Surg Res. 2019;14(1):348.

46. Parvizi J, Diaz-Ledezma C. Total knee replacement with the use of a tourniquet: more pros than cons. Bone Jt J. 2013;95-b(11 Suppl A):133-4.

47. Rama KR, Apsingi S, Poovali S, Jetti A. Timing of tourniquet release in knee arthroplasty. Meta-analysis of randomized, controlled trials. J Bone Jt Surg Am. 2007;89(4):699-705.

48. Worland RL, Arredondo J, Angles F, Lopez-Jimenez F, Jessup DE. Thigh pain following tourniquet application in simultaneous bilateral total knee replacement arthroplasty. J Arthroplasty. 1997;12(8):848-52.

49. Appell HJ, Glöser S, Duarte JA, Zellner A, Soares JM. Skeletal muscle damage during tourniquet-induced ischaemia. The initial step towards atrophy after orthopaedic surgery? Eur J Appl Physiol Occup Physiol. 1993;67(4):342-7

50. Leite CBG, Ranzoni LV, Giglio PN, Bonadio MB, Melo LDP, Demange MK, Gobbi RG. Assessment of the use of tranexamic acid after total knee arthroplasty. Acta Ortop Bras. 2020;28(2):74-7.

51. Drain NP, Gobao VC, Bertolini DM, Smith C, Shah NB, Rothenberger SD, Dombrowski ME, O'Malley MJ, Klatt BA, Hamlin BR, et al. Administration of tranexamic acid improves long-term outcomes in total knee arthroplasty. J Arthroplasty. 2020;35(6s):S201-s206.

52. Morales Santias M, Mas Martinez J, Sanz-Reig J, Martínez Gimenez E, Verdu Román C, Bustamante Suarez de Puga D. Topical tranexamic acid in cemented primary total knee arthroplasty without tourniquet: a prospective randomized study. Eur J Orthop Surg Traumatol. 2020;30(6):1003-8.

53. Hines JT, Petis SM, Amundson AW, Pagnano MW, Sierra RJ, Abdel MP. Intravenous tranexamic acid safely and effectively reduces transfusion rates in revision total knee arthroplasties. J Bone Jt Surg Am. 2020;102(5):381-7

54. Wang K, Ni S, Li Z, Zhong Q, Li R, Li H, Ke Y, Lin J. The effects of tourniquet use in total knee arthroplasty: a randomized, controlled trial. Knee Surg Sports Traumatol Arthrosc. 2017;25(9):2849-57.

55. Fukuda A, Hasegawa M, Kato K, Shi D, Sudo A, Uchida A. Effect of tourniquet application on deep vein thrombosis after total knee arthroplasty. Arch Orthop Trauma Surg. 2007;127(8):671-5.

\section{Publisher's Note}

Springer Nature remains neutral with regard to jurisdictional claims in published maps and institutional affiliations.

Ready to submit your research? Choose BMC and benefit from:

- fast, convenient online submission

- thorough peer review by experienced researchers in your field

- rapid publication on acceptance

- support for research data, including large and complex data types

- gold Open Access which fosters wider collaboration and increased citations

- maximum visibility for your research: over $100 \mathrm{M}$ website views per year

At BMC, research is always in progress.

Learn more biomedcentral.com/submissions 